Trauma Berufskrankh 2014 · 16[Suppl 1]:5-8 DOI 10.1007/s10039-013-2048-0

c) Springer-Verlag Berlin Heidelberg 2014
M. Oberscheven ${ }^{1} \cdot$ A. Kranig ${ }^{2}$

${ }^{1}$ Heilbehandlung/Medizinische Rehabilitation, Deutsche Gesetzliche Unfallversicherung (DGUV), Berlin

${ }^{2}$ Deutsche Gesetzliche Unfallversicherung (DGUV), Berlin

\title{
Neuausrichtung der stationären Heilverfahren
}

\section{Gesetzliche Unfallversicherung}

Mit dem Ziel einer weiteren Stärkung der Effektivität und Effizienz werden die Träger der gesetzlichen Unfallversicherung ihre stationären Heilverfahren beginnend mit dem Jahr 2013 neu ordnen. Die Gremien der Deutschen Gesetzlichen Unfallversicherung e. V. (DGUV) fassten im September 2012 die Beschlüsse zur Neuordnung.

\section{Neue Herausforderungen und veränderte Rahmenbedingungen}

Hintergrund der jetzt eingeleiteten Neuausrichtung in den Heilverfahren sind zum einen weitreichende Veränderungen in den Versorgungsstrukturen insgesamt, zum anderen aber auch steigende Anforderungen an die Qualitätssicherung und spürbare Änderungen im Bedarf der gesetzlichen Unfallversicherung selbst.

Die seit dem Jahr 2003 zu verzeichnenden Entwicklungen in der ärztlichen Weiterbildung mit dem Zusammenwachsen der Fachrichtungen Orthopädie und Unfallchirurgie haben nicht nur eine Veränderung des Qualifikationsprofils der die Verletzten behandelnden Fachärzte zur Folge, sondern darüber hinaus mittelbare und sichtbare Auswirkungen auf die Krankenhausstrukturen und die Organisation der im Bereich der Versorgung von Arbeitsunfallverletzten tätigen Kliniken und Abteilungen. So ist ein Trend hin zu gemeinsamen Zentren und Abteilungen für Orthopädie und Unfallchirurgie zu beobachten, mit der Folge, dass die rein unfallchirurgischen und im Schwerpunkt auf die Notfallversorgung rund um die Uhr ausgerichteten Abteilungen weniger werden und in die Defensive gerieten.

Befördert wird diese Entwicklung durch die mit Einführung des DRG-Fallpauschalensystems (DRG: „diagnosis related groups") geschaffenen Vergütungsstrukturen. Viele Kliniken legen heute den Schwerpunkt verstärkt auf die Durchführung elektiver Eingriffe, was im Hinblick auf Vorhaltekosten und Kapazitätsauslastung deutliche Effizienzsteigerungen verspricht. Eine richtungweisende Antwort auf diese Entwicklung waren die Initiative der Deutschen Gesellschaft für Unfallchirurgie (DGU) zur Bildung von Traumanetzwerken und die damit einhergehende Neuordnung der unfallmedizinischen Landkarte durch das Weißbuch Schwerverletztenversorgung [1], das auch der gesetzlichen Unfallversicherung für ihre Neuordnung der Heilverfahren wichtige Impulse geben konnte.

Mit den jetzt eingeleiteten Schritten zur Neuausrichtung der Heilverfahren sollen zudem die Voraussetzungen geschaffen werden, langfristig neue Wege in der Qualitätssicherung zu beschreiten. Wie alle Zweige der Sozialversicherung ist auch die gesetzliche Unfallversicherung in besonderem Maße gefordert, die Wirksamkeit ihrer besonderen Heilverfahren und auch deren Wirtschaftlichkeit unter Kosten-Nutzen-Aspekten nachzuweisen. $\mathrm{Zu}$ einer modernen und am Bedarf des be- troffenen Menschen ausgerichteten Qualitätssicherung gehört heute zweifelsohne auch die Einbeziehung der Patientenperspektive. Vor dem Hintergrund rückläufiger Gesamtunfallzahlen in der gesetzlichen Unfallversicherung und mit Blick auf die herausgehobene Bedeutung besonders schwerer Verletzungsformen waren die bisherigen Beteiligungsverfahren schließlich insgesamt auf den Prüfstand zu stellen. Die Unfallversicherungsträger stellten sich dabei der Frage, ob sie noch die richtigen Schwerpunkte setzen oder noch stärker als bisher den Fokus auf die schwersten und komplexen Verletzungsarten legen müssen. Denn gerade diese Fälle sind für die Lebensqualität und berufliche bzw. soziale Wiedereingliederung ihrer Versicherten besonders bedeutsam und für die Beitragszahler von Berufsgenossenschaften und Unfallkassen mit besonders hohen Kosten verbunden.

Als Ziele der Neuordnung im stationären Bereich wurden deshalb formuliert:

- eine Konzentration auf die in der Versorgung von Schwerarbeitsunfallverletzten besonders qualifizierten und erfahrenen Kliniken,

- eine stärkere Differenzierung der Heilverfahren nach Art und Schwere der Verletzungsart,

- eine Profilierung und Aktualisierung der Qualitätsanforderungen ein-

Dieser Beitrag erschien ursprünglich in Trauma und Berufskrankheit (2013) 15[Suppl 1]:25-28. DOI s10039-012-1924-3. 
schließlich Schaffung der Voraussetzungen zur Messung von Prozessund Ergebnisqualität sowie

- eine Stärkung der sektorenübergreifenden Versorgung im Sinne der gesetzlichen Unfallversicherung (Alles aus einer Hand - Rehabilitationsmanagement).

\section{Weißbuch Schwerverletztenversorgung}

Mit dem Weißbuch Schwerverletztenversorgung der DGU [1] wurde ab dem Jahr 2006 eine Qualitätsoffensive in der traumatologischen Versorgungslandschaft eingeleitet, die von der DGUV und ihren Mitgliedern von Beginn an konstruktiv begleitet und unterstützt wurde. Die mit klaren Strukturanforderungen hinterlegten, dreistufigen Traumanetzwerke wurden von Anfang an als Vorbild für die unfallversicherungsrechtlichen Heilverfahren angesehen. Hier galt es, Synergieeffekte zu nutzen und die Möglichkeit der Übernahme der von der Fachgesellschaft entwickelten Standards in die Beteiligungsverfahren zu prüfen. Im Ergebnis werden sich die Strukturanforderungen der gesetzlichen Unfallversicherung künftig in Teilen an den Vorgaben des Weißbuchs [1] anlehnen, ohne dass die formale Zertifizierung im Traumanetzwerk $^{\mathrm{D}}$ DGU als notwendige Bedingung für eine Beteiligung durch die DGUV gefordert wird.

Auch strukturell wird die Gliederung des Weißbuchs [1] in der gesetzlichen Unfallversicherung nachvollzogen. So werden die akutstationären Heilverfahren der gesetzlichen Unfallversicherung in Zukunft ebenfalls dreistufig ausgestaltet. Neben dem Durchgangsarzt (D-Arzt) am Krankenhaus und dem bisher für die Versorgung aller schweren Verletzungen vorgesehenen Verletzungsartenverfahren (VAV) wird es künftig ein herausgehobenes Verfahren für die Versorgung schwerster und komplexer Verletzungsmuster in Form des neuen Schwerstverletzungsartenverfahrens (SAV) geben.

\section{Neues stationäres Durchgangsarztverfahren (DAV)}

Für den Durchgangsarzt am Krankenhaus ohne besondere Zulassung zur Versorgung Schwerarbeitsunfallverletzter wird es bedeutsame Veränderungen geben. In diesem Bereich wird die Beteiligung nicht mehr allein durch eine personalisierte $\mathrm{Zu}$ lassung des Arztes zum D-Arzt-Verfahren vollzogen, sondern durch eine Beteiligung des Krankenhauses ergänzt.

Sofern an diesem Krankenhaus unter stationären Bedingungen behandelt werden soll, erfolgt in Zukunft parallel eine vertragliche Zulassung des Krankenhauses anhand klar definierter Strukturanforderungen.

Auf diese Weise werden künftig alle stationären Verfahren in der gesetzlichen Unfallversicherung qualitativ beschrieben und in Beteiligungsverfahren der DGUV überführt. Damit einhergeht im Bereich des DAV eine Anhebung des Behandlungsniveaus, indem der bisherige VAVKatalog in Teilen reduziert wird. Eine Reihe von Verletzungen, z. B. einfache Radiusfrakturen, einfache Sprunggelenkbrüche sowie Sehnenverletzungen, wird künftig nicht mehr im VAV vorgestellt werden müssen. Die DGUV sieht in der Neuregelung des stationären DAV in der beschriebenen Form eine deutliche Aufwertung aller Krankenhäuser, die in relevanter Form Unfallchirurgie betreiben.

\section{Änderungen im Verletzungs- artenverfahren (VAV)}

Im Bereich der Schwerverletztenversorgung wird es ab der Stufe des VAV eine qualitätsorientierte Konzentration geben. Dies wird zum einen durch eine Profilierung der Qualitätsanforderungen erreicht, die sich für den Bereich des VAV am Standard eines regionalen Traumazentrums nach dem Weißbuch der DGU [1] anlehnen werden. Insbesondere für die Verfügbarkeit ärztlicher und nichtärztlicher Mitarbeiter sowie in Bezug auf die Ausstattung der Operationsabteilung, der Notaufnahme und der Intensivstation werden die Vorgaben der Fachgesellschaft in weiten Teilen übernommen.

Aufgrund des unterschiedlichen Versorgungsauftrags im VAV einerseits und im Traumanetzwerk ${ }^{\mathrm{D}}$ DGU andererseits gibt es in einigen Bereichen jedoch auch Abweichungen. Da die Schwerstfälle in der gesetzlichen Unfallversicherung künftig in das neue SAV gesteuert werden sollen, weichen die Anforderungen der DGUV für die Beteiligung am VAV teilweise von den für die endgültige Versorgung auch Schwerst- und Mehrfachverletzter aufgestellten regionalen Traumazentren ab. Mit Blick auf den Versorgungsauftrag der gesetzlichen Unfallversicherung werden außerdem zusätzliche Anforderungen formuliert, die über die im Weißbuch [1] formulierten Standards hinausgehen. Dies betrifft zum einen die Forderung nach einer kindertraumatologischen Kompetenz in den VAV-Kliniken, die durch entsprechende Erfahrungen bei den verantwortlichen Ärzten nachgewiesen werden kann. Darüber hinaus werden die Anforderungen im Bereich der Hygiene um den personellen und organisatorischen Bereich erweitert, wobei ein Verweis auf die Empfehlungen des Robert Koch-Instituts (RKI) erfolgt.

Die Neuregelungen im VAV, die insgesamt eine Anhebung der Versorgungsqualität zum Ziel haben, werden flankiert durch die Wiedereinführung einer Mindestfallzahl. Aus Sicht der gesetzlichen Unfallversicherung ist ein gewisses Mengengerüst durchaus ein aussagekräftiges Indiz für eine hohe Versorgungsqualität und v. a. auch ein Indikator für Routine im Umgang mit den besonderen Verfahrensvorgaben der DGUV. Zum anderen schafft eine gewisse Fallmenge erst die Voraussetzungen, um Qualität messen zu können und aussagekräftige Ergebnisse für die Qualitätssicherung im Bereich der gesetzlichen Unfallversicherung zu erzielen. Nach Abschluss der Analysen wurde die Mindestfallzahl auf 75 VAV-Fälle pro Jahr festgelegt. In einem Betrachtungszeitraum von 5 Jahren muss die Fallzahl entweder im Durchschnitt oder in den letzten 3 Jahren dieses Zeitraumes vorliegen. Entsprechend der regionalen Bedarfslage werden bei drohender Unterversorgung und zur Vermeidung sog. weißer Flecken auf der Landkarte Ausnahmemöglichkeiten von der Mindestfallzahl nach bundeseinheitlichen Kriterien geschaffen. 
Neues Schwerstverletzungsartenverfahren (SAV)

Hierfür sind Verletzungen vorgesehen, die mit lang andauernden Arbeitsunfähigkeitszeiten, einer besonders hohen Rentenrelevanz oder einem hohen Rehabilitationsaufwand verbunden sind. Hier$\mathrm{zu}$ zählen schwerste Verletzungen wie das Polytrauma, Amputationsverletzungen, schwere Schädel-Hirn-Verletzungen, Querschnittlähmungen, aber auch weitere schwerste und komplexe Verletzungen, die aus Sicht der gesetzlichen Unfallversicherung in kompetenten Zentren konzentriert werden sollen.

Die Einführung eines neuen SAV erforderte eine Überarbeitung und Differenzierung des bisherigen Verletzungsartenverzeichnisses, das die Zuweisung der Verletzungsarten in die verschiedenen Versorgungsstufen beschreibt.

Die Anforderungen an die SAV-Kliniken werden sich in weiten Teilen an denen an überregionale Traumazentren gemäß den Vorgaben des Weißbuchs der DGU [1] orientieren. Dies betrifft wiederum die Verfügbarkeit der fachärztlichen Mitarbeiter sowie die Ausstattungsmerkmale der Operationsabteilung, der Notaufnahme und der Intensivmedizin. Darüber hinaus werden Anforderungen an eine möglichst frühzeitig einsetzende, begleitende und multiprofessionelle Rehabilitation sowie an die Kooperation mit dem Rehabilitationsmanagement des verantwortlichen Unfallversicherungsträgers und die Schnittstelle zur weiterführenden Rehabilitation formuliert.

\section{Weiterentwicklung der Netzwerkidee}

Die von der DGU formulierte Traumanetzwerkidee soll mittelfristig für den Bereich der gesetzlichen Unfallversicherung weiterentwickelt werden. $\mathrm{Zu}$ diesem Zweck sollen die Netzwerke um die Bereiche der Rehabilitation und der Behandlung von späten Komplikationen und Rekonstruktionsbehandlungen ergänzt werden. Insbesondere auf der Ebene des SAV sollen deshalb die Voraussetzungen geschaffen werden, die für eine Steuerung und integrierte Versorgung der auf dieser Ebene behandelten, besonders kos-

Trauma Berufskrankh 2014 · 16[Suppl 1]:5-8 DOI 10.1007/s10039-013-2048-0

(c) Springer-Verlag Berlin Heidelberg 2014

\section{Oberscheven · A. Kranig Neuausrichtung der stationären Heilverfahren. Gesetzliche Unfallversicherung}

\section{Zusammenfassung}

Hintergrund. Um die Effektivität und Effizienz der stationären Heilverfahren zu stärken, werden diese ab 2013 neu geordnet. Die Gremien der Deutschen Gesetzlichen Unfallversicherung e. V. (DGUV) fassten im September 2012 die diesbezüglichen Beschlüsse.

Ziele. Als Ziele der Neuordnung im stationären Bereich wurden formuliert: eine Konzentration auf die in der Versorgung von Schwerarbeitsunfallverletzten besonders qualifizierten und erfahrenen Kliniken, eine stärkere Differenzierung der Heilverfahren nach Art und Schwere der Verletzungsart, eine Profilierung und Aktualisierung der Qualitätsanforderungen und eine Stärkung der sektorenübergreifenden Versorgung im Sinne der gesetzlichen Unfallversicherung (Rehabilitationsmanagement).
Umsetzung. Bei der Umsetzung dieser Ziele spielt das Weißbuch Schwerverletztenversorgung der Deutschen Gesellschaft für Unfallchirurgie eine wichtige Rolle. Entscheidende Veränderungen ergeben sich mit dem neuen stationären Durchgangsarzt- und dem neuen Schwerstverletzungsartenverfahren, wobei Letzteres auch mit Änderungen im Verletzungsartenverfahren einhergeht. Auch die Netzwerkidee soll weiter ausgebaut und neue Verfahren der Qualitätssicherung sollen etabliert werden. Erste Änderungen werden bereits am 01.01.2013 in Kraft treten.

\section{Schlüsselwörter}

Unfallversicherung · Schwerverletzte . Verletzungsart · Qualitätssicherung · Rehabilitation

\section{New structure of inpatient treatment. Statutory accident insurance}

\section{Abstract}

Background. In order to increse the effectiveness and efficiency of inpatient treatment a new structure will be implemented in 2013. The committee of the German Statutory Accident Insurance (DGUV) formulated these resolutions in September 2012.

Aims. The aims of restructuring inpatient treatment were formulated as follows: a concentration of the care of severely injured patients after occupational accidents in specially qualified and experienced clinics, an increased differentiation of treatment according to the type and severity of injuries, a streamlining and updating of quality requirements and an increase in interdisciplinary treatment in the sense of statutory accident insurance (rehabilitation management).
Implementation. The White Book on treatment of the severely injured of the German Society for Traumatology played an important role in the implementation of the aims. Decisive amendments include the new accident insurance consultant and the new severe injury procedures whereby the latter also includes amendments to the injury type procedure. The network idea will also be extended further and new procedures for quality assurance will be established. The first changes will come into force on 01.01.2013.

\section{Keywords}

Accident insurance - Severely injured . Injury type - Quality assurance, healthcare . Rehabilitation ten- und folgenträchtigen stationären Fälle notwendig sind.

Die Träger der gesetzlichen Unfallversicherung werden sich daher ab dem Jahr 2013 auch der qualitätsorientierten Neuausrichtung der Rehabilitation Schwerund Schwerstverletzter widmen und die Anforderungen in diesem Bereich ebenfalls überprüfen. Die sektorenübergreifende Netzwerkbildung soll zudem auch inhaltlich durch die Formulierung beson- derer SGB-VII-Rehabilitationsstandards (SGB: Sozialgesetzbuch) definiert werden, wobei ebenfalls v. a. die besonders schweren SAV-Fälle berücksichtigt werden sollen. In Anlehnung an das Phasenmodell im Bereich der neurologischen Rehabilitation sollen damit für weitere schwere und schwerste Verletzungen die Anforderungen in den verschiedenen Phasen der stationären und ambulanten Rehabilitation bis hin zur Nachsorge beschrie- 
ben werden. Ein besonderes Augenmerk gilt dabei den Übergängen zwischen den verschiedenen Rehabilitationsphasen und der Verzahnung mit dem Rehabilitationsmanagement der Unfallversicherungsträger.

\section{Qualitätssicherung in den Heilverfahren}

In einem weiteren Schritt werden die Träger der gesetzlichen Unfallversicherung zu prüfen haben, welche weiteren Maßnahmen der Qualitätssicherung in den stationären Heilverfahren zielführend und umsetzbar sind.

Zweifelsohne gehört hierzu der Einsatz einer patientennahen Ergebnismessung, die die Gesundheit, Lebensqualität und Zufriedenheit der Patienten zum Ziel hat.

Darüber hinaus wird zu untersuchen sein, welche Routinedaten in den Krankenhäusern sich für eine Qualitätssicherung in den Heilverfahren der Unfallversicherung eignen.

Ein besonderes Augenmerk werden die Unfallversicherungsträger künftig auf den Bereich der Prozessqualität, und dabei insbesondere auf das Zusammenwirken von Leistungserbringern und Unfallversicherungsträgern im Rehabilitationsmanagement legen. Wichtig dabei wird sein, dass die im Handlungsleitfaden Reha-Management der DGUV [2] konsentierten Standards der Kooperation und Koordination des Rehabilitationsprozesses im Praxisfall umgesetzt werden.

Im Bereich des SAV schließlich ist die Einrichtung von Qualitätszirkeln geplant, in denen anhand bestimmter klar definierter Qualitätsparameter der Erfolg eines Rehabilitationsprozesses gemessen werden kann und mit denen Qualitätsvergleiche zwischen den beteiligten Leistungserbringern ermöglicht werden sollen.

\section{Inkrafttreten zum 01.01.2013}

Die Anforderungen an die Krankenhäuser im Bereich des DAV, VAV und SAV sowie das neue Verletzungsartenverzeichnis wurden im September 2012 in den verantwortlichen Gremien der DGUV verabschiedet. Die neuen Regeln werden zum 01.01.2013 in Kraft treten. Für den
Aufbau des neuen SAV und die Durchführung der Beteiligungsverfahren durch die DGUV-Landesverbände ist ein Übergangszeitraum von $1 \mathrm{Jahr}$ bis Ende des Jahres 2013 vorgesehen. Zum 01.01.2014 sollen dann auch die für das SAV vorgesehenen Verlegungspfade Anwendung finden und in der Praxis umsetzt werden Für den Bereich des stationären DAV und des VAV ist geplant, dass alle Krankenhäuser in einem Übergangszeitraum von bis zu 5 Jahren nach den neuen Anforderungen überprüft und beteiligt werden.

Schließlich sollen die neuen Heilverfahren Eingang in eine Rahmenvereinbarung zwischen der DGUV und der Deutschen Krankenhausgesellschaft (DKG) über die Krankenhausversorgung von Arbeitsunfallverletzten finden. Nach derzeitigem Stand kann diese Vereinbarung ebenfalls zum 01.01.2013 in Kraft treten.

\section{Korrespondenzadresse}

\section{Oberscheven}

Heilbehandlung/Medizinische Rehabilitation, Deutsche Gesetzliche Unfallversicherung (DGUV),

Mittelstraße 51, 10117 Berlin

markus.oberscheven@dguv.de

Interessenkonflikt. Der korrespondierende Autor gibt für sich und seinen Koautor an, dass kein Interessenkonflikt besteht.

The supplement containing this article is not sponsored by industry.

\section{Literatur}

1. Deutsche Gesellschaft für Unfallchirurgie e. V. (2006) Weißbuch Schwerverletztenversorgung. Thieme, Stuttgart New York

2. Deutsche Gesetzliche Unfallversicherung (2010) Das Reha-Management der Deutschen Gesetzlichen Unfallversicherung - Handlungsleitfaden. DGUV, Berlin 